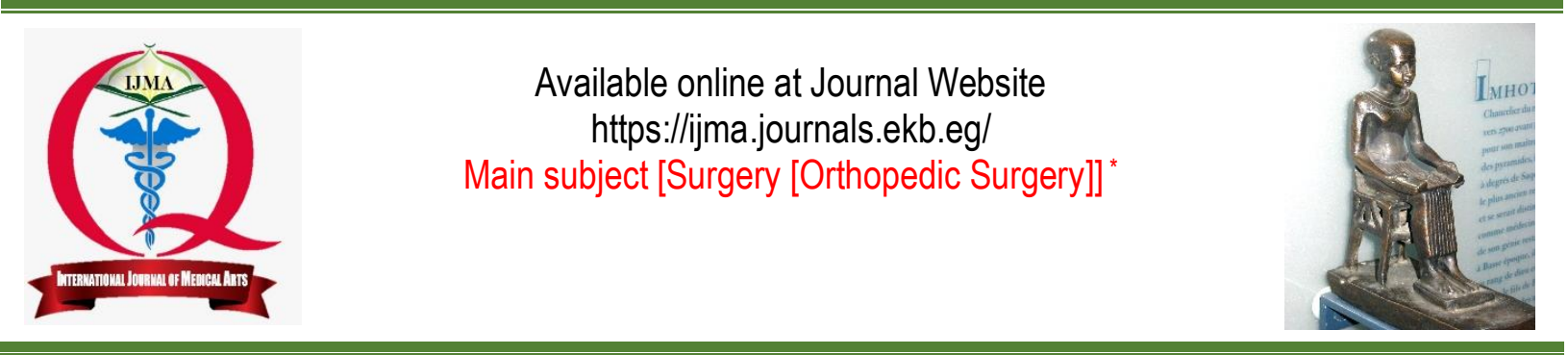

Original article

\title{
Total Knee Arthroplasty for Treatment of Osteoarthritis of the Knee with Severe Malalignment
}

\author{
Osama Mohammed Khalila; Asharaf Ezzedeen ${ }^{\text {b; }}$ Samir Ahmed El-Shourab; Mohamed Abdalah Hassan ${ }^{\text {b }}$ \\ Department of Orthopedic Surgery, Damietta Specialized Hospital, Ministry of Health, Egypt[a]. \\ Department of Orthopedic Surgery, Damietta Faculty of Medicine, Al-Azhar University, Egypt ${ }^{[b]}$.
}

Corresponding author: Osama Mohammed Khalil

Email: dr.osama.khalil78@gmail.com

Received at: January 28, 2020; Revised at: April 17, 2020; Accepted at: April 23, 2020; Available online at: April 24, 2020

\section{ABSTRACT}

Background: Total knee Replacement proved to be an effective surgery that leads to restoration of joint function, limb alignment, beside elimination of pain in patients with osteoarthritis.

Aim of the work: To assess the results of treatment of osteoarthritis of the knee with severe malalignment by total knee arthroplasty.

Patients and Methods: 30 patients [12 males \& 18 females] aged from 48 - 68 years who had advanced knee arthritis were operated by total knee replacement at Al-Azhar University Hospital [Damietta] and Damietta Specialized Hospital and followed up for average 12 months from December 2016 to March 2019. All patients were assessed by history taking, examination, radiological and laboratory investigations, and all were subjected to a three-phase postoperative rehabilitation protocol.

Results: 19 patients [63.3\%] had excellent final result, 8 patients [26.7\%] had good result, 2 patients [6.7\%] had fair result and one patient [3.3\%] had poor final result. In addition, there was significant improvement in pain relief and joint function.

Conclusion: Total Knee Replacement in severe malalignment is a challenging surgery. However, it leads to significant changes in the quality of life in most patients; regarding pain relief, improvement in joint function and early return to daily activities.

Keywords: Knee; Osteoarthritis; Replacement; Malalignment; Outcome.

This is an open access article under the Creative Commons license [CC BY] [https://creativecommons.org/licenses/by/2.0/]

Please cite this article as: Khalil OM, Ezzedeen A, El-Shoura SA, Hassan MA. Total Knee Arthroplasty for Treatment of Osteoarthritis of the Knee with Severe Malalignment. IJMA 2020; 2[2]: 385-398.

* Main subject and any subcategories have been classified according to researchers' main field of study. 


\section{INTRODUCTION}

Osteoarthritis is the most common form of arthritis affecting nearly $30 \%$ of adults over the age of 60 years. Causes of knee arthritis has traditionally been divided into biological and mechanical, although age is a major risk factor for developing arthritis. [1] Varus deformity of arthritic knees is frequently associated with prominent tibial osteophytes and adaptive remodeling of the medial part of the proximal tibia. ${ }^{[2]}$ Smaller metaphyseal defects are reliably managed with bone graft, cement augmented with screw fixation or modular augments. Large metaphyseal defects require more extensive reconstruction such as impaction bone grafting with or without mesh augmentation, prosthetic augmentation, use of bulk structural allograft, or use of metaphyseal wedges, cones or sleeves. ${ }^{[3]}$

Valgus deformity is common in patients with rheumatoid and inflammatory arthropathies and also can occur in those with hypoplastic lateral femoral condyle or previous trauma or reconstructive procedures that change the weight-bearing axis of the lower extremity or tighten the lateral side of the joint. ${ }^{[4]}$ Flexion deformity is not a rare deformity, most preoperative flexion deformities improve with appropriate soft tissue balancing for coronal plane deformity. If a flexion contracture persists despite balanced medial and lateral soft tissues, the shortened posterior structures must be effectively lengthened. Recurvatum deformity is rare in patients who have TKA, reported to be present in less than $1 \%$ of patients. Regardless of the diagnosis for which TKA is indicated [osteoarthritis, posttraumatic arthritis, or inflammatory arthritis], the presence of a recurvatum deformity presents a unique situation. [4] Total knee arthroplasty has become a successful procedure for treating end stage osteoarthritis because of its effectiveness in relieving arthritic pain, correcting deformities, and restoring function. The continued success of total knee arthroplasty is due to improvement in surgical technique, bearing surfaces and implant design. [5] Primary total knee arthroplasty [TKA] is generally a successful procedure, but the life span of the prosthesis is finite. Patients may require revision TKA due to mechanical wear, aseptic loosening, infection, instability or malalignment among other reasons. Implant positioning is closely linked to outcome in primary and revision TKA, and the criteria for an acceptable result include restoration of physiological joint line position and correct implant component position in sagittal, coronal and transverse planes. ${ }^{[6]}$ Wound condition after primary total knee arthroplasty is important for the prevention of periprothetic infection. ${ }^{[7]}$

\section{AIM OF THE WORK}

The aim of this work is to assess the results of treatment of osteoarthritis of the knee with severe malalignment by total knee arthroplasty.

\section{PATIENTS AND METHODS}

Thirty patients [ 12 males \& 18 females] aged from 48 - 68 years who had advanced knee osteoarthritis were operated by total knee replacement at Al-Azhar University Hospital [Damietta] and Damietta Specialized Hospital and followed up for average 12 months from December 2016 to March 2019. All were assessed clinically by history taking, physical examination [General and local systematic knee examination] and preoperative radiological evaluation [Standing A-P standing view of the knee, Lateral view of the knee, Skyline view of the knee, and A-P standing Long leg film]. In addition, a short form of knee society score was used to assess the knee and patient's functional abilities such as walking and stair climbing before and after operative intervention. This scoring system has been validated and applicable across sex, age, activity level, and implant type. The scoring system is available through [http://www.kneesociety.org]. The questionnaire is divided into sections; the first for demographic data, the second to assess symptoms [mainly pain] the third for patient satisfaction; the fourth for functional activities; the fifth for standard activities, and the last for discrepancy activities.

Preoperative preparation included good control of any systemic disease, lab investigations [complete blood count, bleeding time, clotting time, fasting blood sugar, liver \& kidney functions], good preoperative hydration and one dose of the antibiotic that had been used after surgery [usually $3^{\text {rd }}$ generation cephalosporin]. Antibiotic was given within one hour before surgery.

\section{Surgical technique:}

The majority of patients had spinal anesthesia, 6 of them had combined spinal-epidural. They were operated in supine position with body supports on 
both sides. A stopper was fixed to the table to support the limb with the knee in at least $90^{\circ}$ flexed [Figure 1]. All bony prominences were padded and protected to avoid any pressure sores during surgery. A tourniquet was used after limb exsanguination at the junction of the upper third and lower two thirds of the thigh.

Limb preparation: It is started the night before surgery and the patient is advised to have a shower. Cleaning with soap and water followed by a topical antiseptic. Sterile disposable drapes and gowns were used [Figure 2]. Shaving was done only in hairy persons just before entry to the operating theatre, while the patient is still in the induction room.

Approach: The incision was made from a point one hand-breadth above the superior pole of the patella to the inferomedial aspect of the tibial tuberosity [Figure 3A]. The skin, fat, and fascia are incised directly down to the extensor mechanism, and the medial and lateral flaps are gently reflected [Figure 3B]

Arthrotomy: The arthrotomy was done using the medial parapatellar approach in all cases. The quadriceps tendon was cut longitudinally from proximal to distal along its medial border, leaving a cuff of tendon approximately 5 to $10 \mathrm{~mm}$ wide. Then the incision was carried out distally along the medial border of the patella and patellar tendon. The arthrotomy incision was made through the medial retinaculum, capsule, and synovium, leaving a $5-\mathrm{mm}$ cuff of retinaculum attached to the patella to facilitate repair at the end of the procedure. [Figure 4].

Knee joint exposure: The medial side of the knee was exposed by subperiosteally elevating the anteromedial capsule and deep medial collateral ligament. The patella was everted and lateral patellofemoral plicae were released, and then the knee was flexed. Small Hohmann retractors were used to protect the collateral ligaments. [Figure 5].

Preparation of the femur: An anterior synovectomy was performed to expose the supracondylar region of the femur. This was essential to allow for precise sizing of the femoral component and to prevent notching. IM alignment guide was used for preparation of the femur in all cases. A drill was used to make a starting hole in the intercondylar notch for the intramedullary guide. It was placed at the intercondylar notch $1 \mathrm{~cm}$ above the femoral origin of the PCL with drill parallel to the femoral shaft with low speed to avoid notching or perforation. [Figure 6]. Suction of the medullary canal was done before inserting the intramedullary alignment rod to remove the canal content. This allowed decompressing the femoral canal and subsequently reducing the risk of fat emboli. The intramedullary guide was inserted directly in the center of the canal without making any contact with the femoral cortices. The cutting block was attached to the intramedullary rod, adjusted at 5 to 6 degrees of valgus, and then was fixed in place [Figure 7-A]. The thickness of distal femoral cut was doublechecked using the angle wing-measuring guide. The intramedullary rod was removed and the distal femoral cut was finished. [Figure 7-B].

Other cuts of the femur: AP sizing guide was used to make 3 degrees of external rotation of the femoral component in relation to the posterior condylar axis. The sizing stylus should be on the highest point of the anterior cortex to avoid notching. [Figure 8]. After sizing, 2 holes were drilled in the distal femur marking the rotation of the sizer. The sizer was then removed with attaching of the femoral cutting device after marking and determination of the femur size and cuts were done as follows: Anterior and Posterior Femoral Condyle Cuts. Anterior and Posterior chamfer Cuts. Femoral notch "since all cases are posterior stabilized implant". [Figure 9].

The ACL was excised, allowing anterior translation of the tibia. This was aided by external rotation of the tibia. A retractor was applied to gently retract the tibia forward so that exposure of the posterior tibial plateau was achieved [Figure 10]. The medial and lateral menisci and PCL were excised. Osteophytes were excised using bone nibbler.

Bone preparation of the tibia: Extra medullary guide was used in all cases. The upper platform was aligned with the medial third of the tibial tuberosity and the medial margin of the lateral tibial spine. The arms of the platform were positioned against the anterior tibial cortex. The tibial stylus was then used to determine the exact level of resection. Then the tibial alignment handle was assembled to the cutting block and the long alignment rod was passed. It should align with the center of the ankle. The proximal tibia resection guide was fixed in place with 2 pins. The proximal tibia then resected while the knee kept in flexion, 2 retractors also kept in place to 
protect the collateral ligaments [Figure 11]. With the knee in maximal flexion, the tibia was subluxed anteriorly, using retractor; the appropriate tibial tray was selected that most completely covers the cut surface without over-hanging or under-hanging after removing of the osteophytes. Following size selection and adjusting the rotation the tray is fixed to the proximal tibia by 2 threaded pins. Drilling the proximal tibia done using the specific guide drill for the selected tibia size. Tibial Broach was inserted and taped once to be seated [Figure 12]. Now, the preparation and bone cuts of both femur and tibia are both finished.

Trial reduction and soft tissue balancing: The femoral trial was positioned on the prepared femoral surface using the femoral inserter. Then an appropriate trial tibial tray was chosen and impacted in place using special applicator. Care was taken that mal-rotation can occur and was cautious to avoid it. The corresponding plastic trial insert is placed on to the trial tibial tray. [Figure 13]. Then the knee is reduced and the range of motion was checked in flexion and full extension. Examination of the knee for medial and lateral stability as well as overall alignment in the coronal and sagittal planes in full extension took place. The extension space is the gap that is formed after distal femur and proximal tibia are resected, the extension gap. The flexion space is the gap that is formed after the posterior femur and the proximal tibia are resected, the flexion gap. These spaces should be rectangular in configuration. Where they are trapezoidal, the medial and lateral soft tissues must be balanced. In severe varus knees, proximal tibial periosteum was released along the tibial brim around to the posterior medial corner. Then, if needed extended release to further fibers of the MCL was done. In cases with bone defects less than $5 \mathrm{~mm}$ bone cement was used to fill the defect after drilling 2-mm holes in the sclerotic floor of the defect. [Figure 14].

In cases with bone defects more than $5 \mathrm{~mm}$ bone graft from the resected cuts was used after removing all sclerotic avascular bone and fixed with screws. [Figure 15]. In larger defects metal wedges were used.

In severe Valgus knees, release of the lateral structures took place while minimizing medial dissection. In general, the sequence of the release was the lateral collateral ligament, iliotibial band, popliteus tendon, and lateral capsular structures. In severe flexion contracture, posterior osteophytes were excised. When needed, posterior capsule from posterior femur was released. In cases when flexion contracture persisted, the distal femoral bone cut was increased by $2 \mathrm{~mm}$ and re-checked to see if the knee moved into full extension with the trial components in place.

Patellar procedure: Patellar osteophytes were removed with smoothening the edge of the patella [patelloplasty] [Figure 16].

Implant Fixation: For the whole study, a cemented PS [posterior-cruciate substituting] implant was used، after trial and balancing the knee, the cement was mixed and we flushed the knee with normal saline, cleaning and drying the knee by suction followed by cementing the implant. Trial spacer was used to press the implant for proper cementing and after hardening of the cement we used sequence of trial spacers to check the proper spacer size [Figure 17]. Again, after the final spacers, checking the balance of the knee and ligaments integrity done with the range of motion.

Hemostasis and Closure: The tourniquet was routinely deflated by the end of the operation and meticulous hemostasis was carried on before wound closure. Suction drains were used in all cases and applied deep to the capsular closure in the lateral and medial gutters. Meticulous closure of the arthrotomy was performed by interrupted figure of eight sutures using [Vicryl No.1] with the knee in mild flexion. The subcutaneous layer was closed with particular attention to the superficial fascia overlying the quadriceps tendon, using vicryl NO. 20 . The skin was closed with simple interrupted sutures [Figure 18].

Postoperative medications: Analgesia; if the patient had an epidural catheter, a single dose of analgesic was enough. If no epidural catheter was available, good analgesia was mandatory in the form of pethidine $50 \mathrm{mg}$ three times daily for two days, then continued by non-steroidal anti-inflammatory drugs twice daily for five days then oral if needed only. Antibiotics in the form of 3rd generation cephalosporin, parenteral for two days then five days' oral in non-complicated cases. All cases received enoxaparin $40 \mathrm{mg}$ prefilled syringe subcutaneously once daily for 14 days. 
Postoperative follow-up: Patients with bilateral knee replacement were usually discharged between day 7- day10 postoperative according to their general conditions, progress with the physiotherapy and the surgical wound conditions. This period was extended in one patient who developed superficial wound infection [which responds to conservative treatment by antibiotics and standard wound cares]. Patient with unilateral knee replacement stayed shorter [4-6 days]. All patients of the study were followed up at 15, 21, 30 and 60 Days. Postoperatively, they underwent postoperative $X$ ray short film at the 15th Days follow up visit and long leg standing $X$ ray at 30th day follow up visit [used for measuring the study results]. Non-complicated patients were asked for a regular follow up visits at 6-month intervals.

Rehabilitation: Post-operative rehabilitation protocol consisted of three phases:

Phase I: Immediate Post-Surgical Phase [Day 03]. Active/active assisted/passive [A/AA/PROM] exercises seated and supine. Isometric quadriceps, hamstring, and gluteal isometric exercises. Straight leg raises [SLR]. Lower extremity range of motion and strengthening. Gait training on flat surfaces as tolerated by the patient. Cryotherapy $1-3 x /$ day for swelling and pain management.

Phase II: Motion Phase [Day 3-Week 6]. AA/A/PROM, stretching for flexion [ $>90$ degrees] and full extension. Continue isometric quadriceps, hamstring, and gluteal isometric exercises. Supine heel slides and seated Long Arc Quad [LAQ]. Straight Leg Raising [SLR]. Gait training to improve function and quality of involved limb performance during swing through and stance phase.

Phase III: [weeks 6-12]: Continue exercises listed in Phase II with progression including resistance and repetitions.

Methods of statistical analysis: Data were fed to the computer and analyzed using IBM SPSS software package version 20.0. Qualitative data were described using number and percent. Quantitative data were described using mean, standard deviation for parametric data after testing normality using Kolmogrov-Smirnov test.

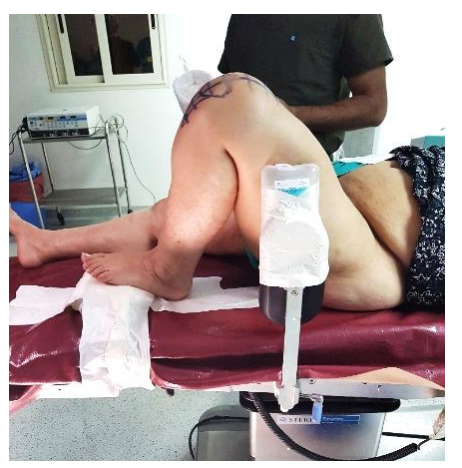

Figure [1]: Operative photo showing side support and stopper.

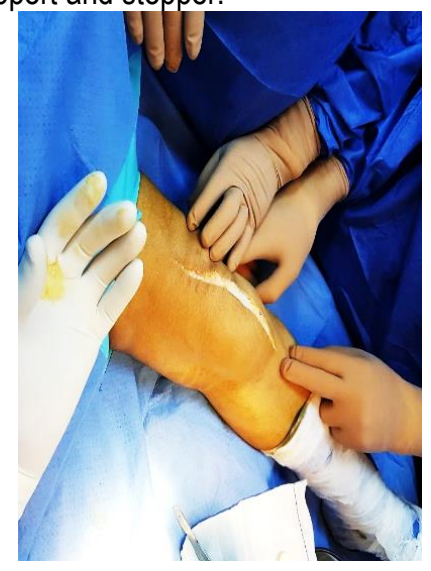

A

Figure [3]: Operative photos showing: [A] Skin incision, [B] Developing skin and subcutaneous tissue flaps.

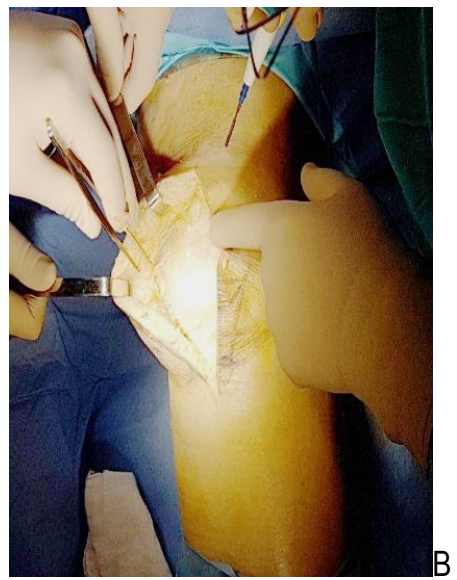

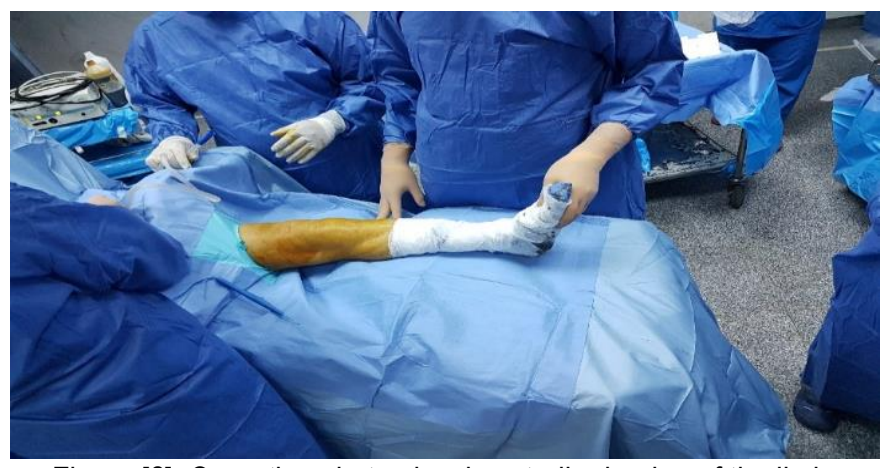

Figure [2]: Operative photo showing sterile draping of the limb.

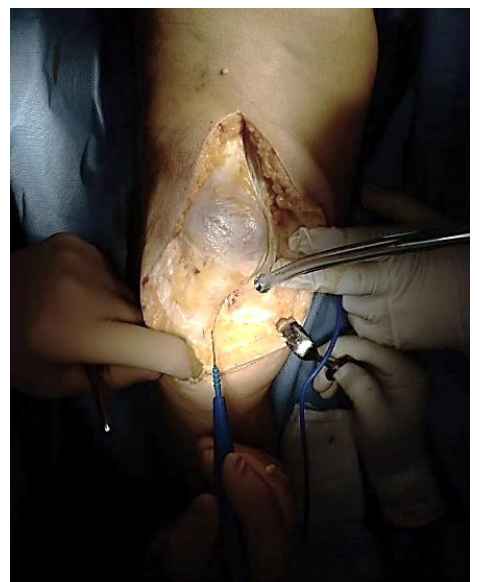

Figure [4]: Operative photo showing arthrotomy through medial parapatellar approach. 


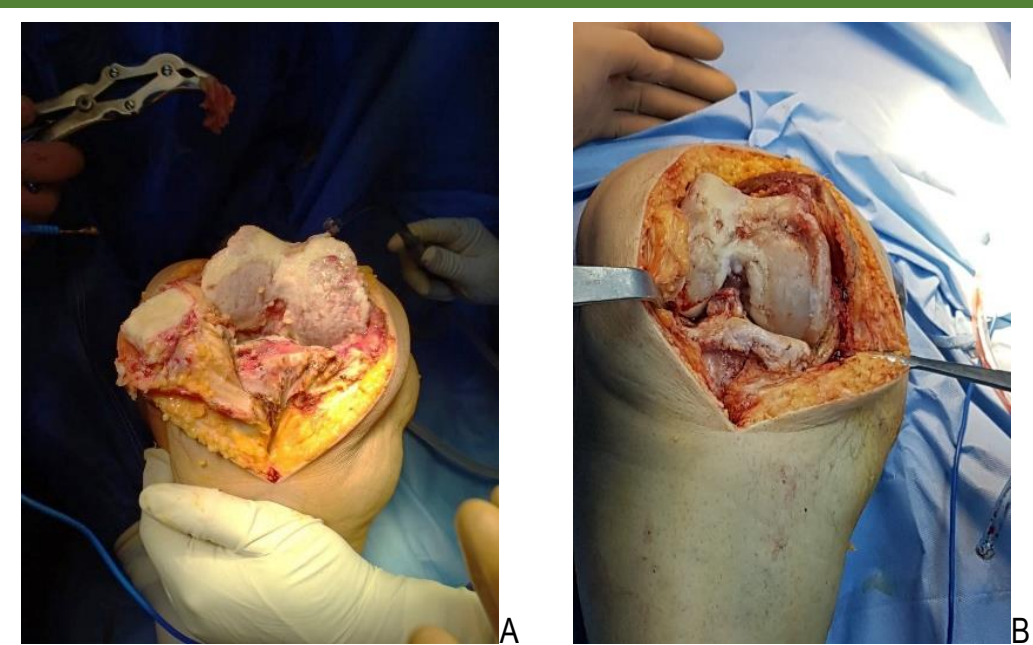

Figure [5]: Operative photos showing: $[\mathrm{A}]$ exposure of distal femur and patellar eversion, $[\mathrm{B}]$ Hohmann retractors used to protect collateral ligaments.
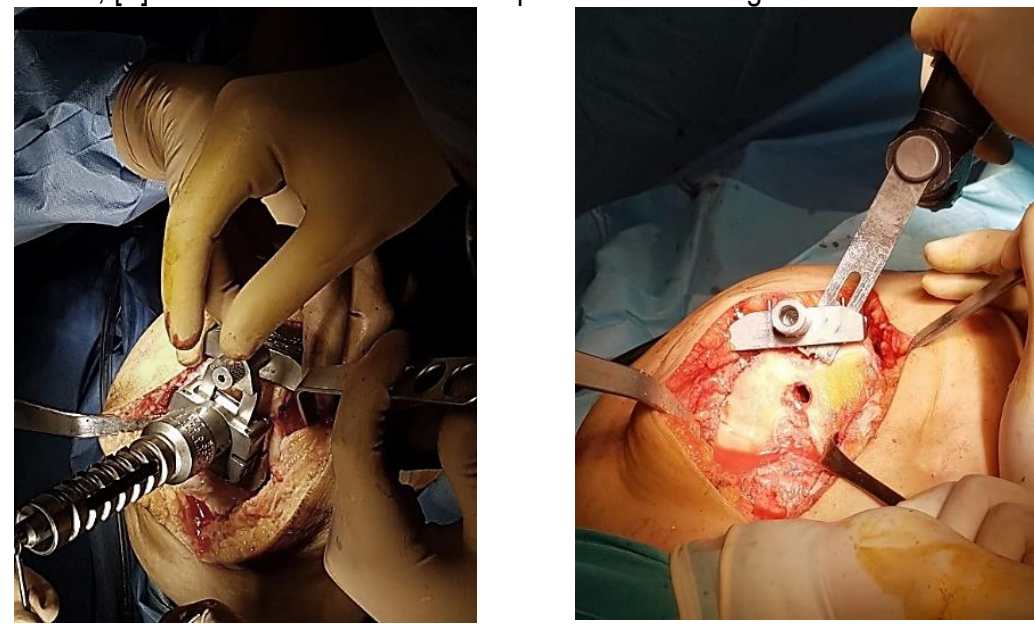

Figure [7]: Operative photos showing: [A] Cutting block attached to the intramedullary rod. [B] Distal femoral cut with electric saw.

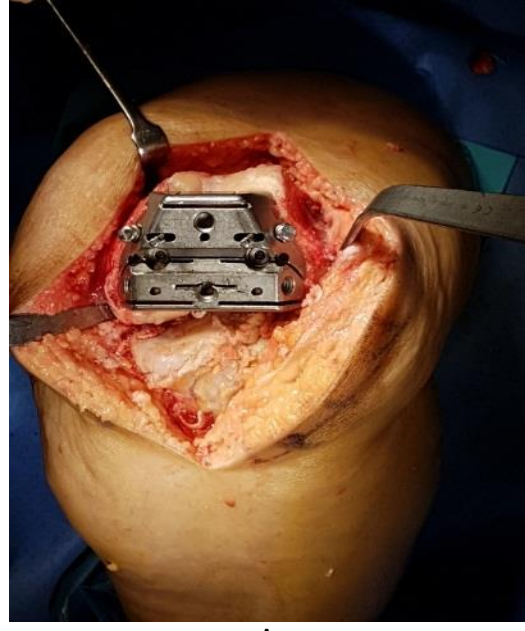

A

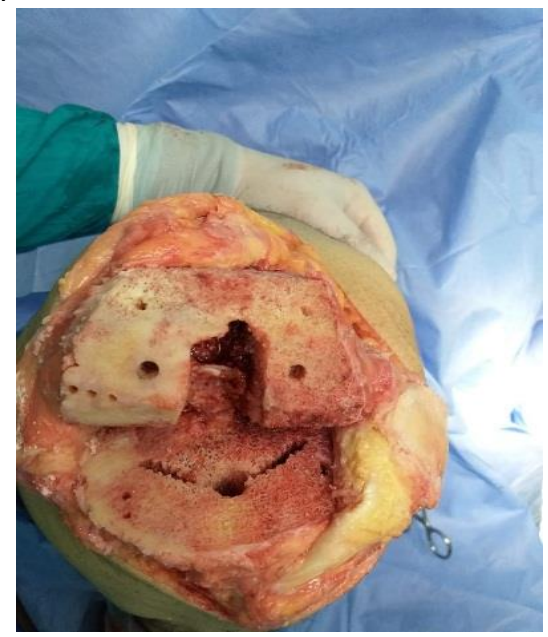

$B$
Figure [9]: Operative photos showing: $[A]$ Cutting block to complete femoral cuts. $[B]$ Distal femoral cut finished.

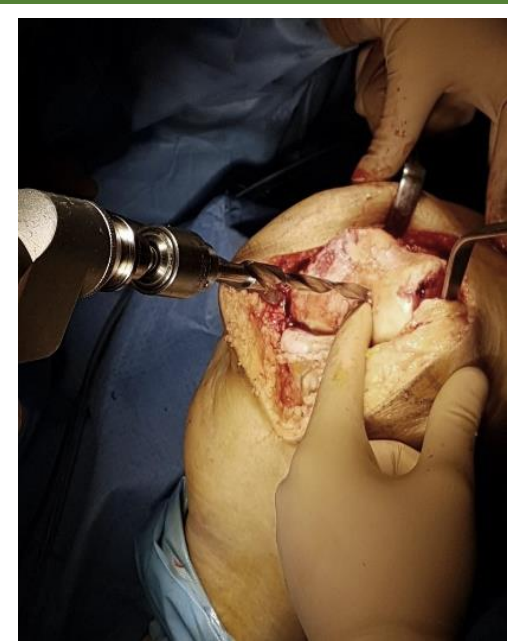

Figure [6]: Operative photo showing opening medullary canal of the femur.

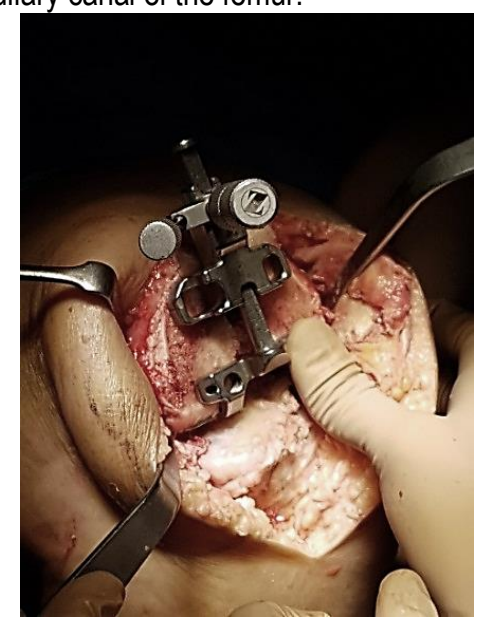

Figure [8]: Operative photo showing sizing of the femur

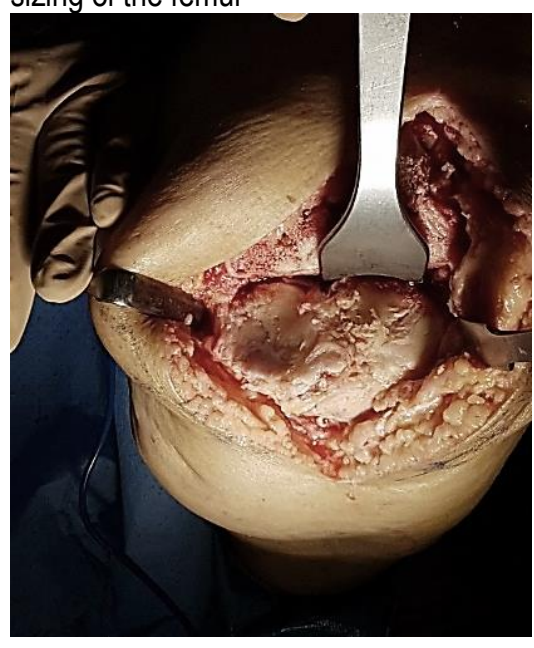

Figure [10]: Operative photo showing using retractor to expose the posterior tibial plateau. 

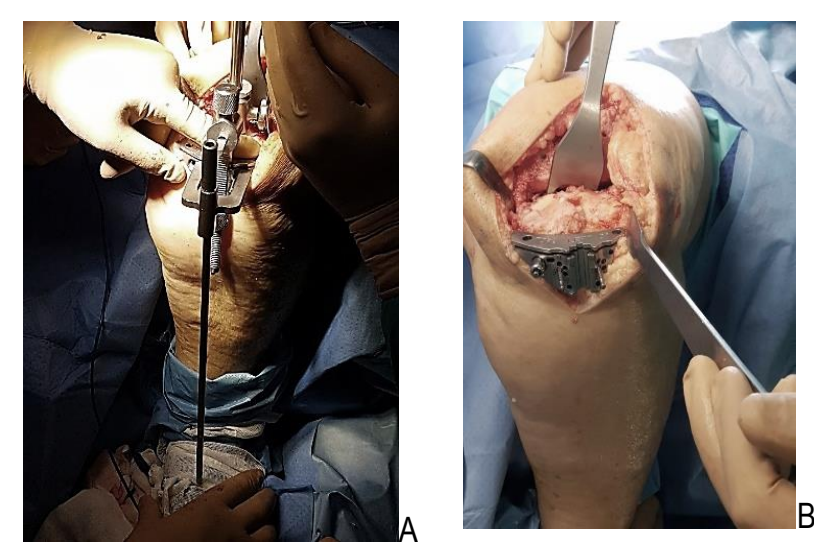

Figure [11]: Operative photos showing: [A] Extra medullary tibial guide. [B] Proximal tibia resection guide fixed in place.

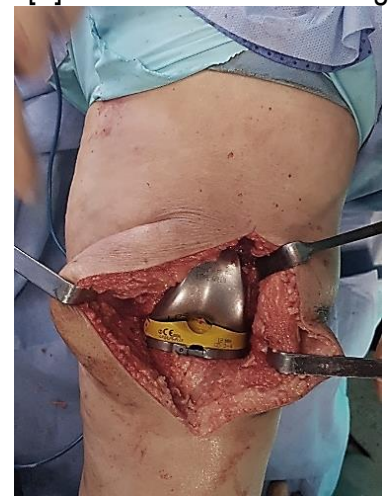

Figure [13]: Operative photo showing trial reduction.

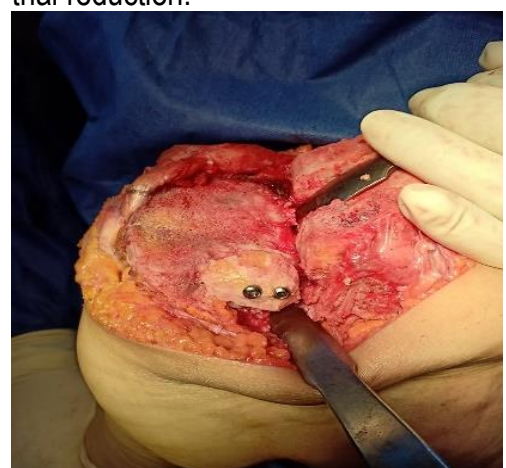

Figure [15]: Operative photos showing medial bone defect filled with bone graft fixed by 2 screws.

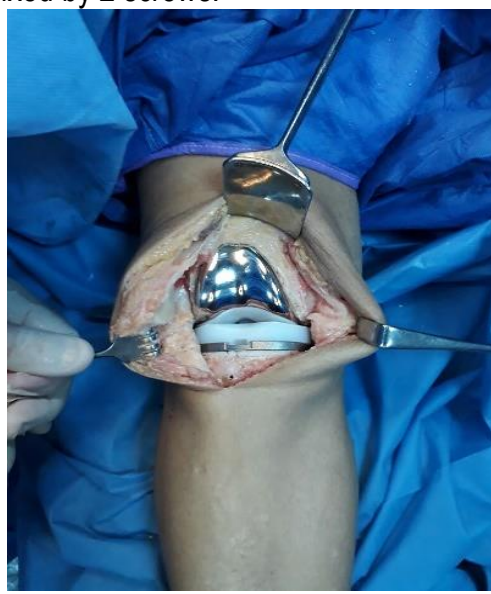

Figure [17]: Operative photos showing final implant fixation. with bone cement.

A drain.
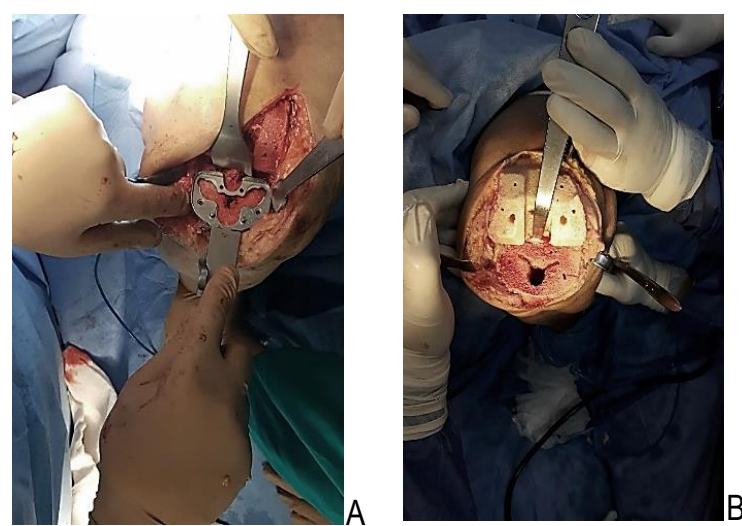

Figure [12]: Figure [12]: Operative photos showing: [A] Sizing of the tibia. [B] Proximal tibia resection and reaming.

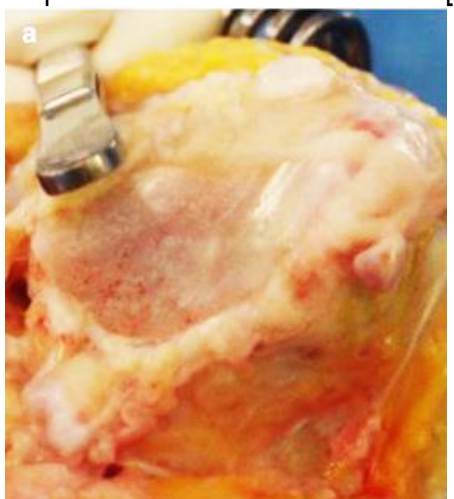

Figure [14]: Operative photos showing: $[\mathrm{A}]$ Medial bone defect. [B] Filling the defect
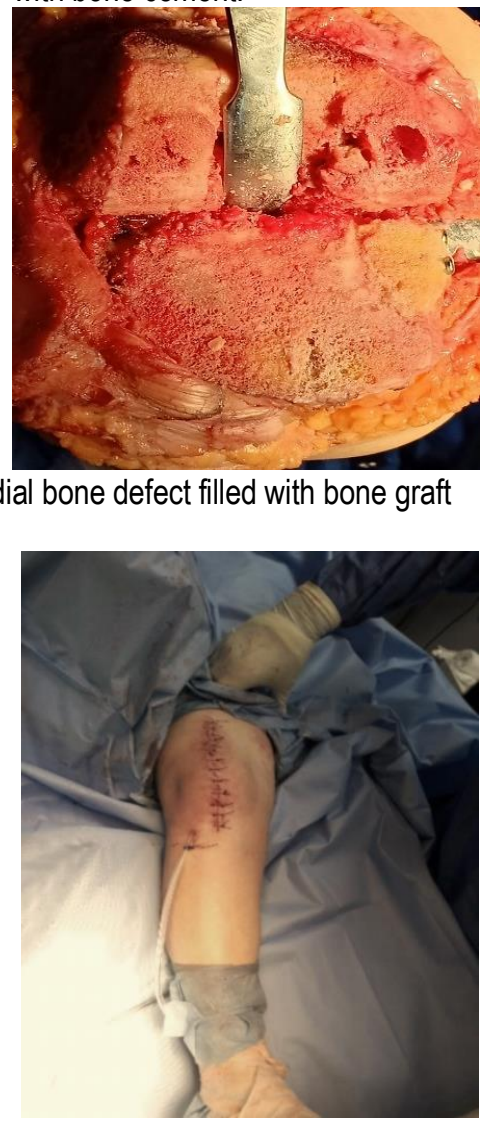
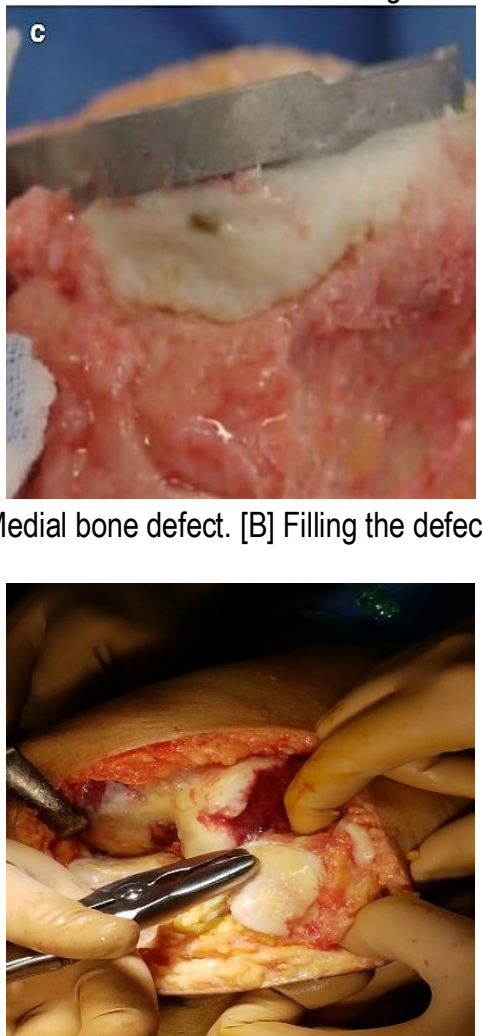

Figure [16]: Operative photos showing removal of patellar osteophytes.

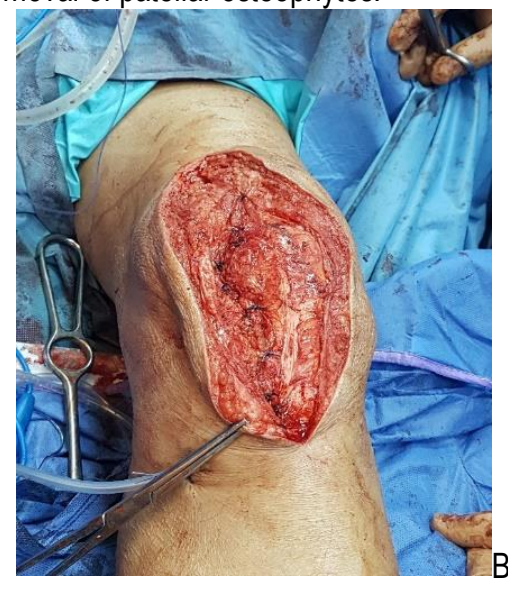

Figure [18]: Operative photos showing: $[A]$ Arthrotomy closure. [B] Skin closure and 


\section{RESUTLS}

In this study, the patients were evaluated after follow up for 12 months-average according to knee society score [KSS] based on the following: Pain, Flexion Contracture, Extension lag, Total Range of Flexion, Alignment [Varus \& Valgus], Stability [Antero-posterior, Medio lateral] and function [walking, stairs, walking aids used].

In the present work, pain was significantly decreased at the last follow up visit when compared to preoperative values $[20.0 \pm 5.8$ vs $40.0 \pm 10.8$ respectively]. On other side, range of motion, anteroposterior stability and mediolateral stability were significantly increase at the last follow up visit when compared to preoperative values [Table 1].

After follow up period the results of this work after pre-operative and post-operative assessment was 19 patients [63.3\%] had excellent final result, 8 patients [26.7\%] had good final result, 2 patients [6.7 $\%$ ] had fair final result and one patient [3.3 \%] had poor final result [Table 2]. The final results showed significant increase of surgery time in fair outcome when compared to good, poor or excellent outcome $[155.0 \pm 7.1$ vs $130.0 \pm 0.0,140.0 \pm 16.9$ and $120.0 \pm$ 16.3 respectively]. However, no significant association was reported between patient age and final outcome [Table 3].

In the present work, no significant relation was reported between patient gender and final results or between deformity and final results [Table 4]. There was statistically significant increase of KSS at final follow up visit when compared to preoperative values [80.70 \pm 7.71 vs $45.13 \pm 5.20$ respectively] [Table 5].

Table [1]: Pre-operative and post -operative mean pain distribution among studied cases.

\begin{tabular}{|l|c|c|c|c|c|c|}
\hline \multirow{2}{*}{} & \multicolumn{2}{|c|}{ Preoperative } & \multicolumn{2}{c|}{ Final } & Paired & P \\
\cline { 2 - 7 } & Mean \pm SD & Min-Max & Mean \pm SD & Min-Max & & \\
\hline Pain & $40.0 \pm 10.8$ & $20-50$ & $20.0 \pm 5.8$ & $0-30$ & 34.4 & $<0.001^{*}$ \\
\hline ROM & $17.5 \pm 2.5$ & $10-20$ & $21.0 \pm 3.6$ & $15-25$ & 17.9 & $<0.001^{*}$ \\
\hline Anteroposterior stability & $5.45 \pm 2.8$ & $0-10$ & $7.9 \pm 0.92$ & $5-10$ & 8.9 & $0.02^{*}$ \\
\hline Mediolateral stability & $6.85 \pm 1.9$ & $0-10$ & $11.8 \pm 0.89$ & $10-15$ & 17.8 & $0.003^{*}$ \\
\hline
\end{tabular}

Table [2]: Distribution of the studied cases according to final results.

\begin{tabular}{|l|c|c|}
\hline \multicolumn{1}{|c|}{ Final results } & $\mathrm{N}=30$ & $\%$ \\
\hline Poor & 1 & 3.3 \\
Fair & 2 & 6.7 \\
Good & 8 & 26.7 \\
Excellent & 19 & 63.3 \\
\hline
\end{tabular}

Table [3]: Relation between final results and age \& surgery time among the studied cases

\begin{tabular}{|c|c|c|c|c|c|}
\hline & $\begin{array}{c}\text { Poor } \\
\mathrm{n}=1\end{array}$ & $\begin{array}{c}\text { Fair } \\
\mathrm{n}=2\end{array}$ & $\begin{array}{c}\text { Good } \\
\mathrm{n}=8\end{array}$ & $\begin{array}{c}\text { Excellent } \\
\mathrm{n}=19\end{array}$ & Test of significance \\
\hline Age/years & $66.0 \pm 0.0$ & $61.0 \pm 5.66$ & $60.0 \pm 5.78$ & $59.84 \pm 6.13$ & $\mathrm{~F}=0.35 ; \mathrm{P}=0.79$ \\
\hline Surgery time/ minutes & $130.0 \pm 0.0$ & $155.0 \pm 7.1$ & $140.0 \pm 16.9$ & $120 \pm 16.3$ & $\mathrm{~F}=4.85 ; \mathrm{P}=0.008^{*}$ \\
\hline
\end{tabular}

Table [4]: Relation between final results and sex of the studied cases

\begin{tabular}{|c|c|c|c|c|c|c|}
\hline \multicolumn{2}{|c|}{} & $\begin{array}{c}\text { Poor } \\
\mathrm{n}=1[\%]\end{array}$ & $\begin{array}{c}\text { Fair } \\
\mathrm{n}=2[\%]\end{array}$ & $\begin{array}{c}\text { Good } \\
\mathrm{n}=8[\%]\end{array}$ & $\begin{array}{c}\text { Excellent } \\
\mathrm{n}=19[\%]\end{array}$ & Test of significance \\
\hline \multirow{2}{*}{ Sex } & Male & $1[100.0]$ & $0[0.0]$ & $2[25.0]$ & $9[47.4]$ & $\mathrm{MC}$ \\
& Female & $0[0.0]$ & $2[100.0]$ & $6[75.0]$ & $10[52.6]$ & $\mathrm{p}=0.26$ \\
\hline \multirow{2}{*}{ Deformity } & Valgus & 0 & $1[50.0]$ & $2[25.0]$ & $4[21.1]$ & $\mathrm{MC}$ \\
& Varus & $1[100.0]$ & $1[50.0]$ & $6[75.0]$ & $15[78.9]$ & $\mathrm{P}=0.76$ \\
\hline
\end{tabular}

Table [5]: pre-operative and post -operative mean KSS distribution among studied cases

\begin{tabular}{|l|c|c|}
\hline & Mean \pm SD & Min-Max \\
\hline Pre-operative KSS & $45.13 \pm 5.20$ & $37.0-55.0$ \\
\hline Final KSS & $80.70 \pm 7.71$ & $59.0-90.0$ \\
\hline \multicolumn{2}{|c|}{$\mathrm{t}=21.7 ; p<0.001^{*}$} \\
\hline
\end{tabular}


Case presentation: A 62-year-old male patient with positive medical history with bilateral advanced knee osteoarthritis. He had Total Knee Replacement using PS implant. Knee society score for the right knee improved from 39 to 73 at final follow up with good outcome, while knee society score for the left knee improved from 38 to 81 at final follow up with excellent outcome [Figure s19-30].
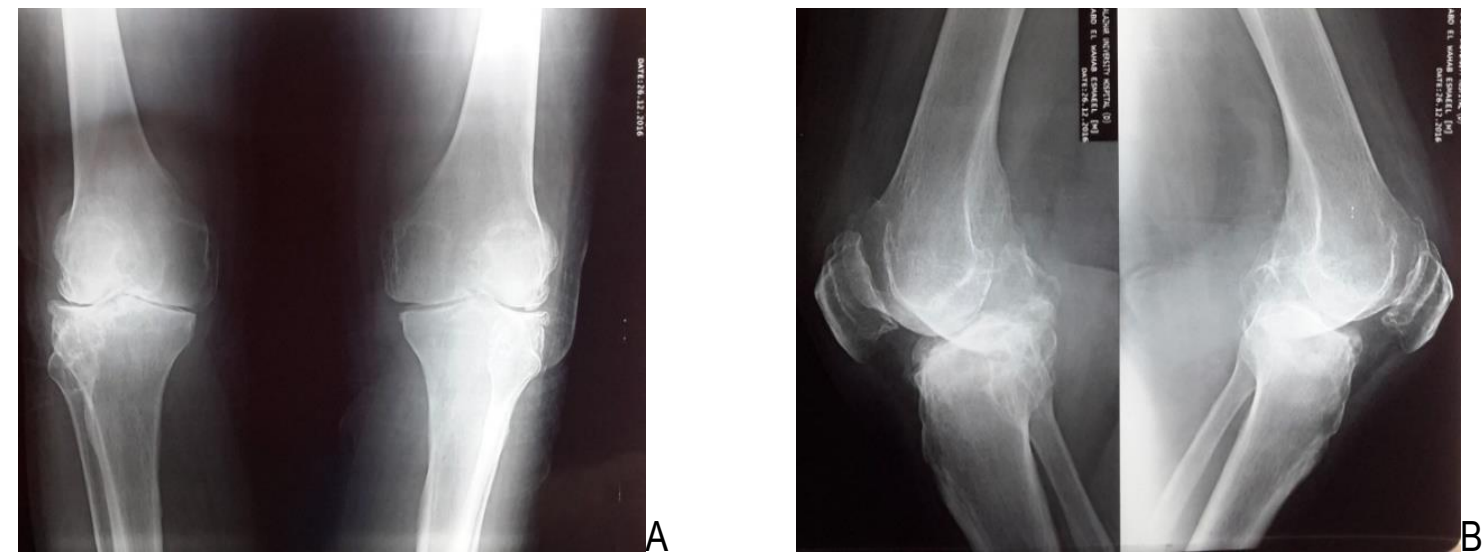

Figure [19]: showing preoperative $[A]$ : anteroposterior view of both knees; $[B]$ : lateral view of both knees
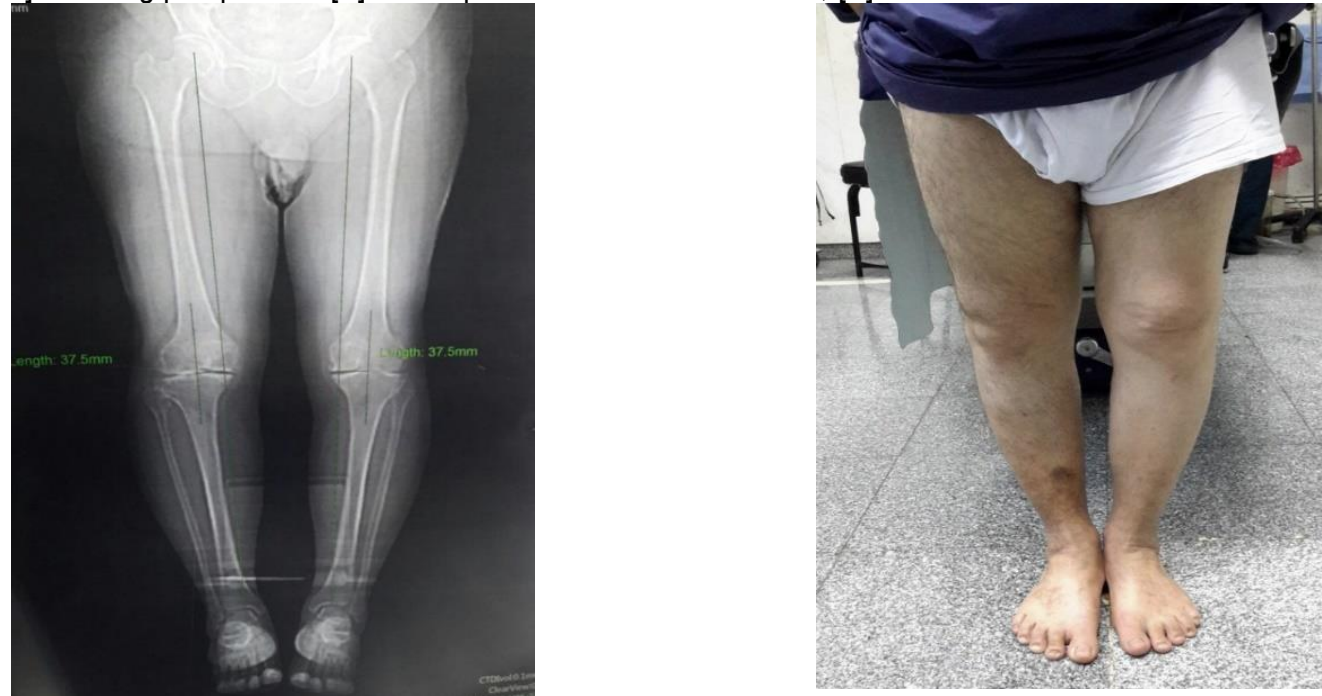

Figure [20]: showing preoperative full-length standing x-ray of both lower limbs

Figure [21]: showing clinical pre-operative standing front view of both knees

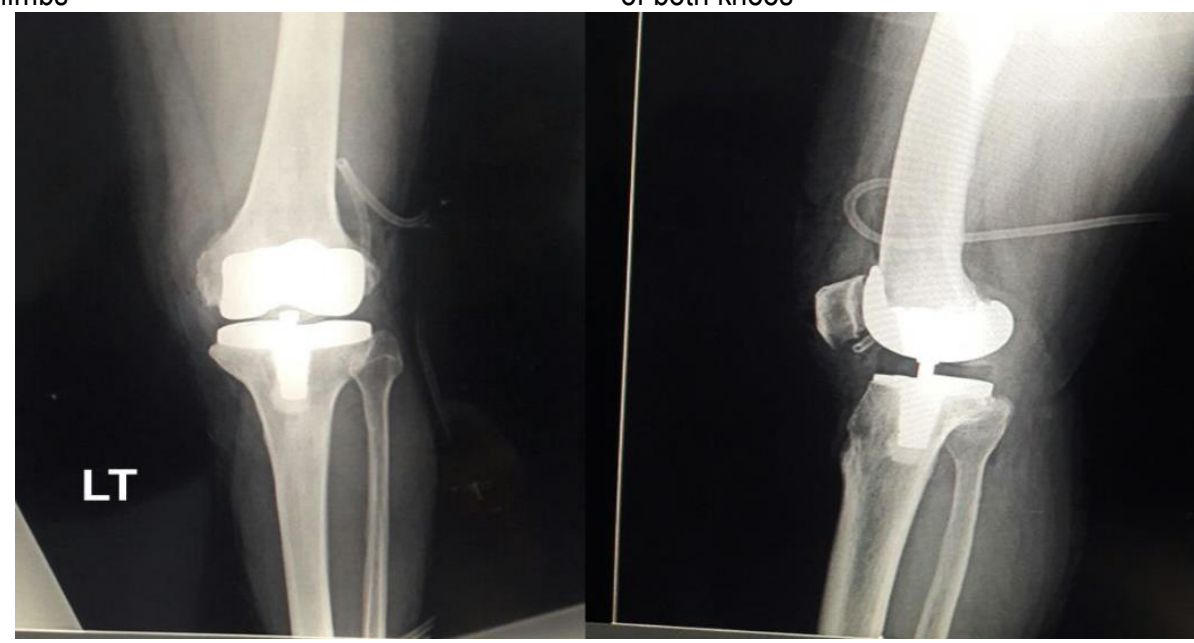

Figure [22]: showing immediate postoperative x-ray of left knee using PS implant. 


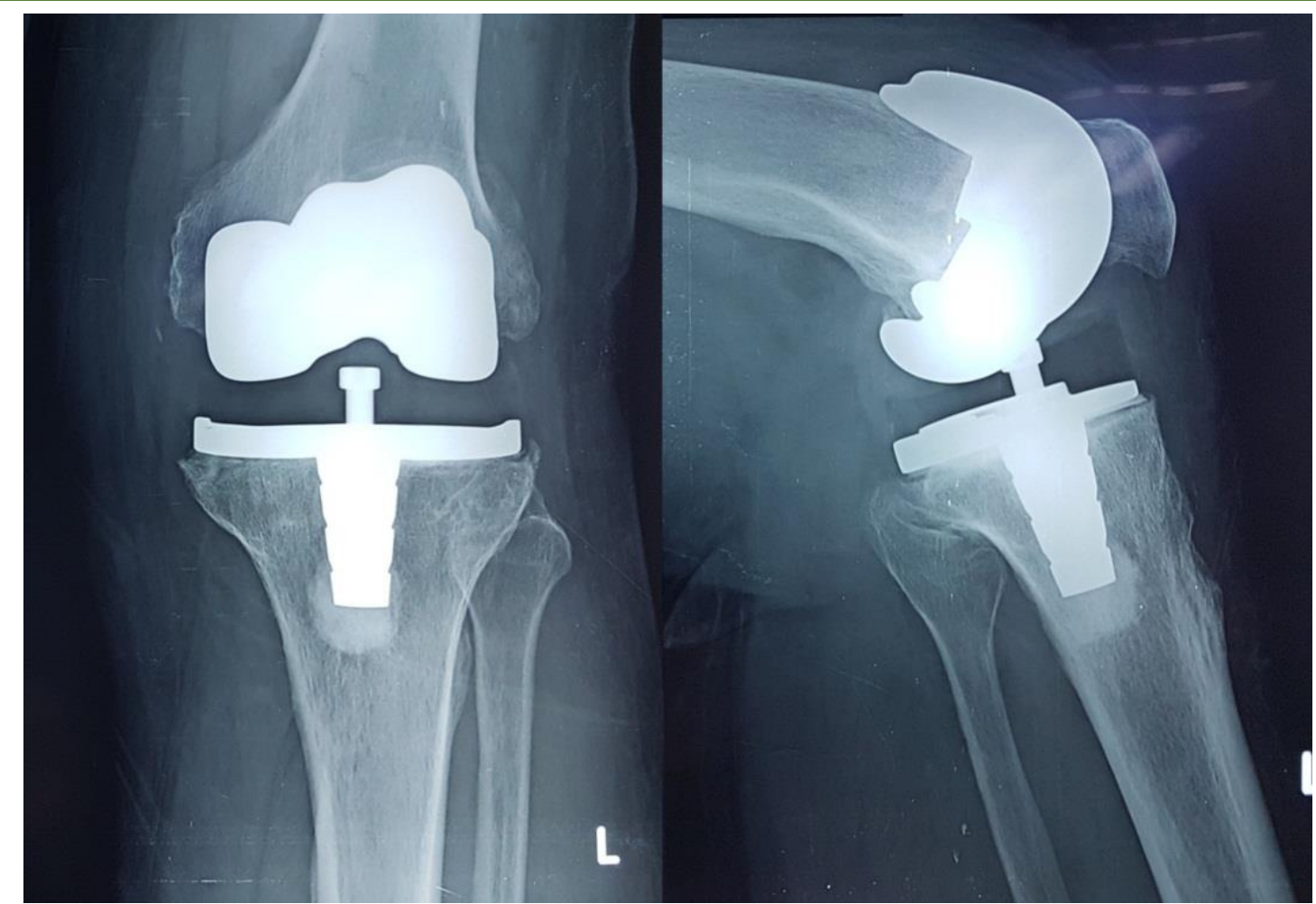

Figure [23]: showing 3-month follow up x-ray of left knee
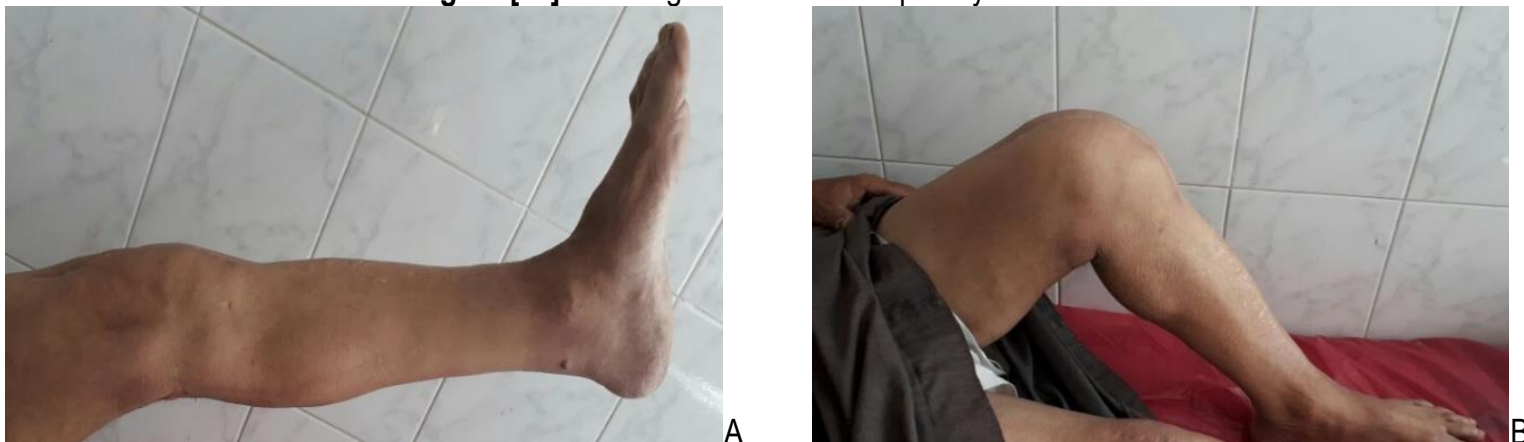

Figure [24]: showing clinical 3-month-follow up side views of left knee [A]: extension [B]: flexion
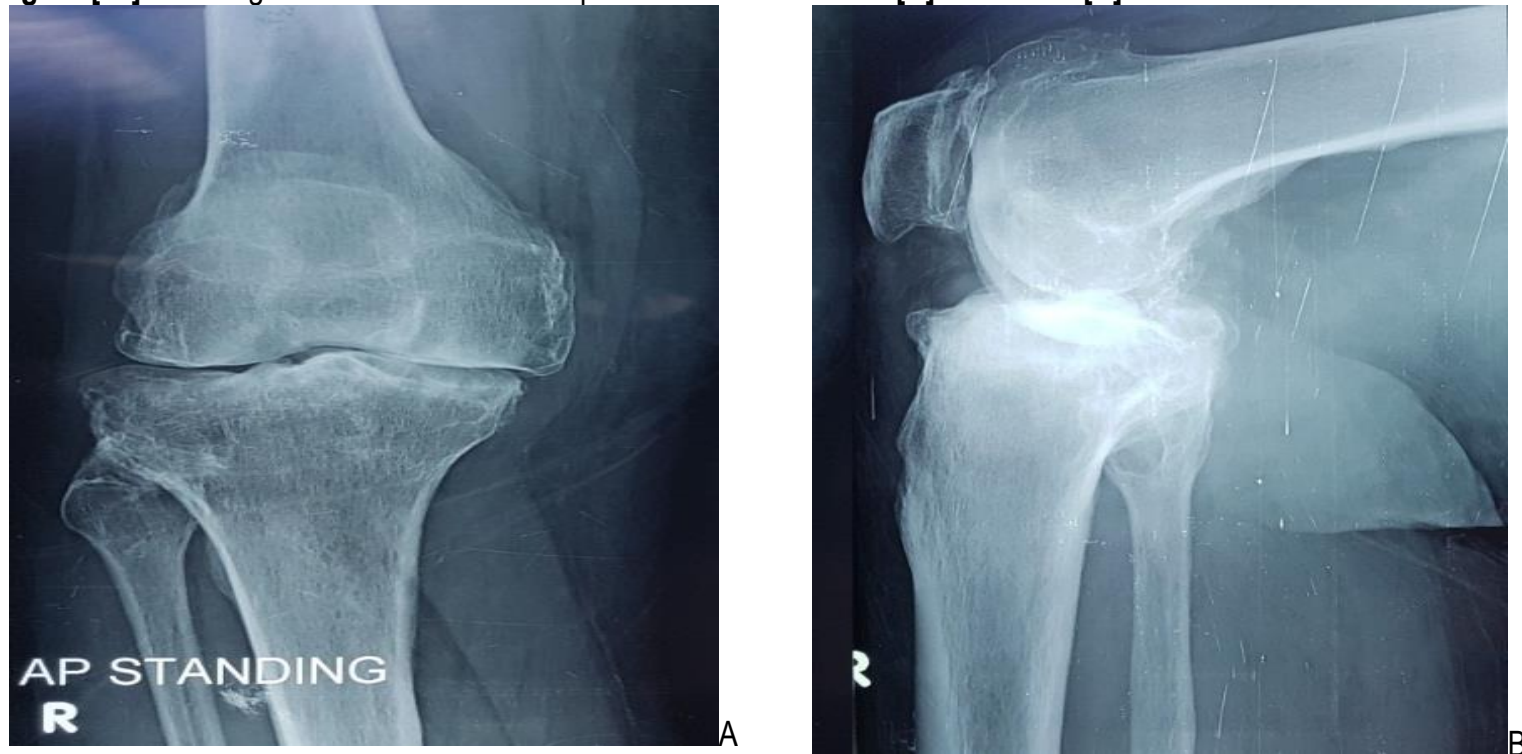

Figure [25]: showing preoperative $x$-ray of right knee $[\mathrm{A}]$ : anteroposterior [B]: lateral 


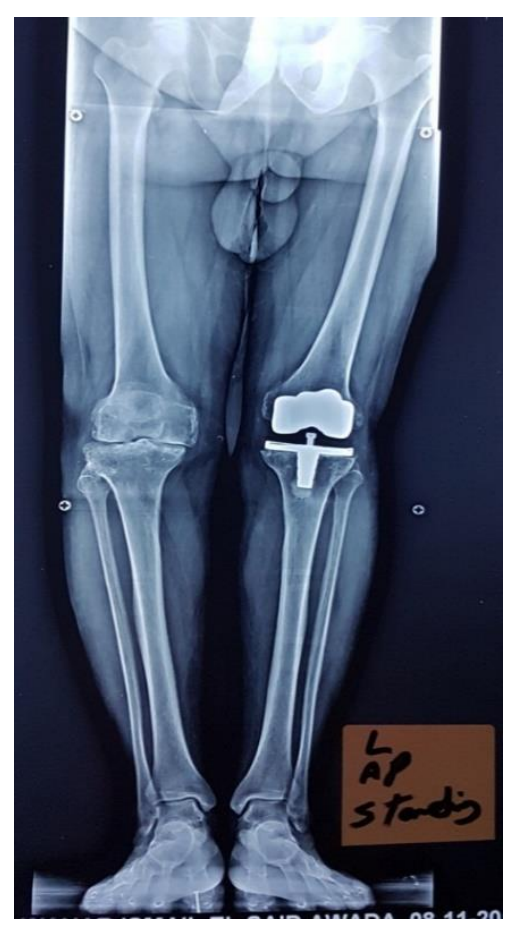

Figure [26]: showing preoperative full-length standing x-ray of both lower limbs with preoperative view of right knee and 11-month follow-up of left knee

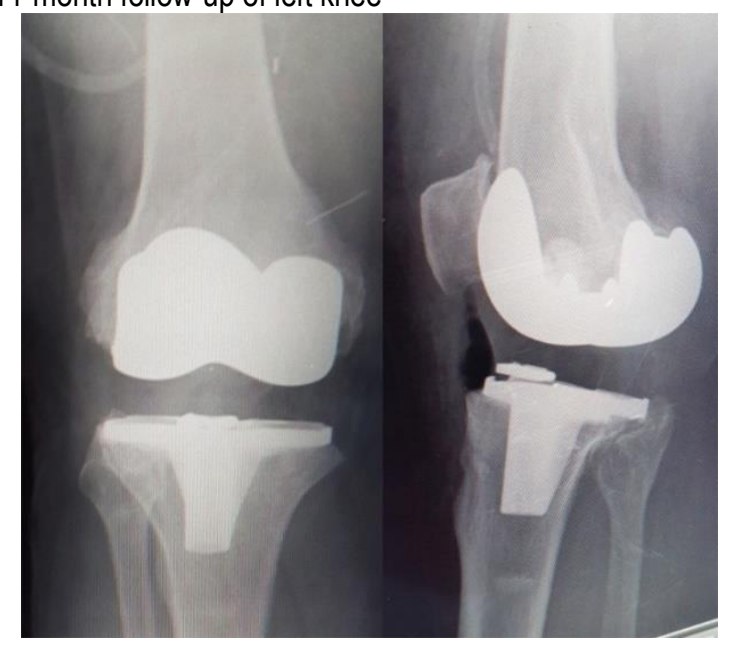

Figure [28]: showing immediate postoperative x-ray of right knee using PS implant.

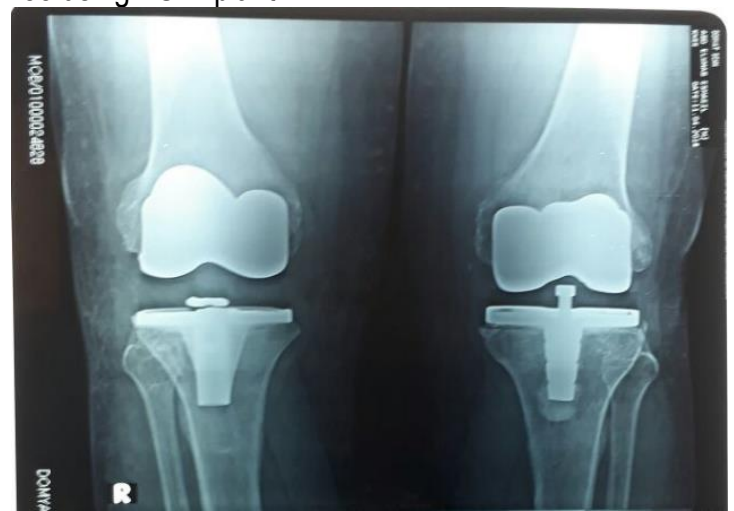

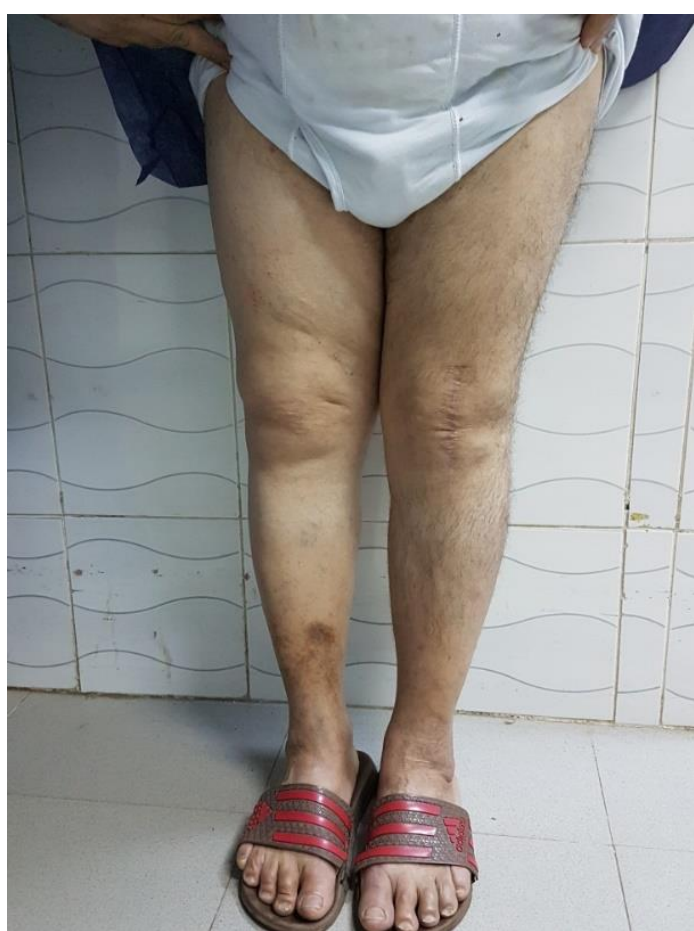

Figure [27]: showing clinical preoperative standing front view of right knee and follow up of left knee
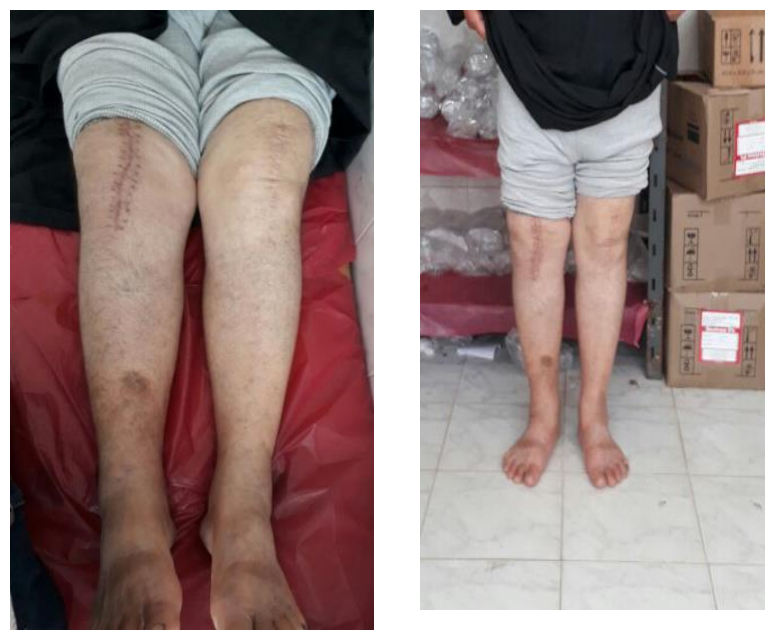

Figure [29]: showing clinical views of both knees [A]: supine [B]: standing

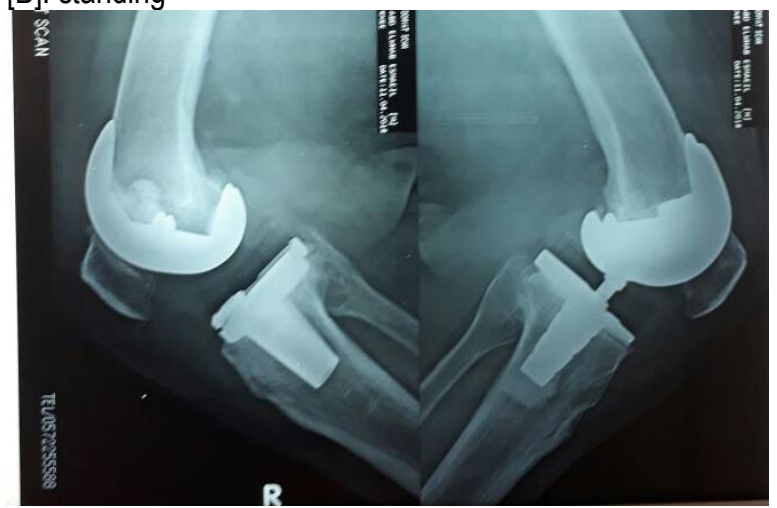

Figure [30]: showing 1-year follow-up or right knee 


\section{DISUCSSION}

Arthritic knees with severe deformities have always constituted a problem from a surgical and a financial point. There are different methods described to correct severe deformity during knee arthroplasty. However, there are few reports in the literature dealing with large numbers of highly deformed knees. [8]

In the current study, 23 cases with knee varus [others 7 had valgus deformity] ranged from $15^{\circ}$ to $20^{\circ}$ were operated by TKR with a posterior stabilized prosthesis. Their age ranged from $55-68$ years. The mean KSS improved from 44.86 preoperatively to 80.69 postoperatively. Varus was corrected via proximal tibial periosteum release along the tibial brim around to the posterior medial corner in all cases. Release of the fibers of the MCL in only 5 cases. Hyun et al. ${ }^{[9]}$ performed a prospective randomized controlled study on 27 patients [40 cases] with $\geq 10^{\circ}$ tibio-femoral varus deformity on the preoperative whole leg standing anteroposterior [AP] view. All study participants were female with a mean age of 71.5 years [range, 62 to 83 years]. The mean preoperative, anatomical tibio-femoral axis was varus $13.3^{\circ}$ [range, $11^{\circ}$ to $1^{\circ}$ ]. They performed TKR using posterior stabilized type prosthesis in all cases. For ligament balancing, the conventional medial soft tissue release was performed in 20 cases [medial release group]. In the remaining 20 cases [vertical osteotomy group], a vertical osteotomy for one size smaller tibial component was performed using an osteotome in the proximal medial tibia for ligament balancing. ${ }^{[9]}$

There was no statistically significant difference in age and preoperative varus deformity angle, range of motion [ROM], and hospital for special surgery [HSS] score between the groups. In the medial release group, the knee joint was exposed using a midvastus approach and medial soft tissue was released up to $1 \mathrm{~cm}$ distally from the subperiosteal layer of the joint capsular insertion site, on the proximal tibia and then posteromedially. Progressive soft tissue release was carried out until symmetrical; medial-lateral balance was confirmed using a trial prosthesis after femoral and tibial articular surface resection. ${ }^{[9]}$

The initial medial release was extended $\geq 1 \mathrm{~cm}$ distally in all of the medial release group [ $n=20]$. Medial-lateral tension was assessed with the knee in extension and $90^{\circ}$ flexion. If medial soft tissue contracture was noted in extension, the deep layer of the MCL and the posteromedial joint capsule were released; whereas the superficial layer of the $\mathrm{MCL}$ and the anteromedial joint capsule were released, if contracture was noted in $90^{\circ}$ flexion to obtain a perfect ligament balance. [9]

In the vertical osteotomy group, a midvastus approach was used for joint exposure. Soft tissue release was started in the subperiosteal layer of the joint capsular insertion site on the proximal tibia and extended $1 \mathrm{~cm}$ distally and posteromedially. Subsequently, without further release, femoral and tibial articular surface resection was carried out and ligament balance was assessed using trial prosthesis. [9]

Taking care to achieve symmetrical mediallateral tension with the use of a prosthesis that is one size smaller than the trial one, an osteotome was inserted perpendicular to the resected articular surface for bone tissue removal in the proximal medial tibia. The bone resection was performed in 2 $\mathrm{mm}$ increments according to the level of the medial soft tissue contracture until a perfect ligament balance was confirmed with the insertion of trial prosthesis. ${ }^{[9]}$

Comparisons between the groups were based on the total operation time, ROM, HSS score, tibiofemoral medial lateral gap ratio examined with the knee in $00^{\circ}, 90^{\circ}$, and $130^{\circ}$ flexion under an image intensifier at 6 months postoperatively. On the assessment of ROM at postoperative 6 months, the difference in the angle created by the femoral longitudinal axis and the tibial longitudinal axis with the knee in maximum flexion and extension on lateral radiographs was recorded. [9]

They found statistically significant intergroup differences were found in the total OP time and the tibio-femoral medial-lateral gap ratio in $130^{\circ}$ flexion. There was no significant difference in the preoperative HSS score and tibio-femoral angle on the whole leg standing AP view and postoperative ROM, HSS score, and tibiofemoral medial-lateral gap ratio in $0^{\circ}$ and $90^{\circ}$ flexion. The mean total OP time was remarkably short in the vertical osteotomy group [mean, 96.9 minutes; range, 80 to 119 minutes] compared to that in the medial release group [mean, 116.2 minutes; range, 109 to 125 minutes] [ $p=0.000$ ]. The mean tibio-femoral medial lateral gap ratio in 
$130^{\circ}$ flexion at postoperative 6 months was notably smaller in the vertical osteotomy group [1.02] than in the medial release group [1.14; $p=0.000]$. However, the ratio was not significantly different between the groups in 00 and 900 flexion [9]

No significant intergroup difference was found in the ROM and HSS score at postoperative 6 months. In the vertical osteotomy group, the tibial component size was determined after bone resection by assessing the medial-lateral tension with a trial prosthesis. In this group, the final prosthesis of choice was one size smaller than the one selected before bone resection, which did not result in a size mismatch with the femoral component. In the medial release group, there were 2 cases of intraoperative partial MCL tear, which did not lead to any clinical problems after staple fixation. ${ }^{[9]}$

In the current study, 16 cases with knee flexion deformity ranged from $10^{\circ}$ to $15^{\circ}$ were operated by TKR with a posterior stabilized prosthesis. Their age ranged from $55-68$ years. The mean KSS improved from 43.75 preoperatively to 74.12 postoperatively. Flexion was corrected via excision of posterior osteophytes in all cases. We released posterior capsule from posterior femur in 7 cases. We increased the distal femoral bone cut by $2 \mathrm{~mm}$ in 5 cases.

Salam et al. ${ }^{[10]}$ performed a retrospective study reviewed severe flexion contracture of patients who underwent primary TKA and soft tissue balancing. All patients were diagnosed as arthritis of knee. The inclusion criteria were knees had flexion contracture with valgus deformity.

Exclusion criteria included pathologic conditions of the knee [trauma, tumor, or infection]. Four patients initially fulfilled the study criteria. According to the criterion of flexion contracture which is beyond or less $30^{\circ}$. patients available for analysis were divided into two groups, i.e., severe flexion group [SF] [with flexion contracture beyond $30^{\circ}$; 4cases initially, and moderate flexion group [MF] [with flexion contracture less $30^{\circ}$ and beyond $10^{\circ} ; 4$ cases initially. ${ }^{[10]}$

There was no significant difference between two groups $p>0.05]$. The TKA surgical procedure included a standard anteromedial approach, the use of an intramedullary femoral and extra medullary tibial alignment rod, measured bone resection, and differential ligament balance in flexion and extension. Measured resection implies that the amount of bone resected from the intact compartment of the joint equals the thickness of the implant, while restoring correct alignment by resecting the bone perpendicular to the mechanical axis. ${ }^{[10]}$

Based on the correct osteotomy, recovering full extension at the end of surgery is mandatory, by first releasing the posterior capsule and the collateral ligaments from their osteophytes and secondly by extending the distal femoral cut where necessary. Once the correct bony alignment is achieved, the flexion and extension spaces are secured equally without massive soft tissue release and an additional distal femur cut. [10]

It is very important in procedures not only to restore a balance between the osteotomy and ligament release but also to maintain the joint tension to prevent joint laxity in severe flexion contracture of knees. ${ }^{[10]}$

In the current study, 7 cases with knee valgus ranged from $10^{\circ}$ to $15^{\circ}$ were operated by TKR with a posterior stabilized prosthesis. Their age ranged from 48 - 67 years. The mean KSS improved from 46 preoperatively to 80.71 postoperatively.[10] Valgus was corrected via release of the lateral collateral ligament, iliotibial band, popliteus tendon, and lateral capsular structures in all cases. [11]

Tucker et al..[11] performed a consecutive series of 275 predominantly cementless TKRs in 262 patients were performed for severe valgus $\left[\geq 10^{\circ}\right]$ deformity and prospectively followed to 10 years. Patient reported outcome measures included the Oxford Knee Score, American Knee Society score, Bartlett Patellar score and the Short Form 12 questionnaire.

Average valgus deformity was reduced from 15.6 to 3.8 degrees [ $p<0.001]$. At a mean follow up of 10.4 years [range 9.5-14.1], 90 [34.4\%] patients had died. Of the reviewed survivors the mean Oxford Knee Score was 27.8+/-9.8, with an American Knee Society clinical score of $85.6+/-17.0$ and a functional score of $65.1+/-20.4$, with $78 \%$ of patients reporting good to excellent results. [11]

A medial approach was used in all knees and following a neutral tibial cut, the anteroposterior distal femoral cuts were made followed by a conservative distal femoral pre-cut of $5^{\circ}$. The 
extension gap was then measured using a spacer block and if balanced [ $<1 \mathrm{~mm} 88$ difference] a definitive distal femoral cut was made in $5^{\circ}$. If unbalanced [trapezoidal extension gap] and the difference between 2 and $5 \mathrm{~mm}$ the definitive distal femoral cut was made in $6^{\circ}$ of valgus or more so as to balance the gap. If $\geq 6 \mathrm{~mm}$ a posterolateral capsular release was performed by placing the knee in full extension with a laminar spreader to open the lateral joint space. The lateral border of popliteus locates the tight posterolateral capsule which when cut over a distance of $10 \mathrm{~mm}$ produces a visual opening. This corrects both fixed flexion and valgus deformity. [11]

The limitation of this study is the short follow-up and relatively small number of cases compared with article on primary knee arthroplasty, but severe deformities are less common, and the early results are promising, although there is a need for a longer follow-up. ${ }^{[8]}$

Conclusion: Total Knee arthroplasty in severe malalignment is associated with significant improvement, regarding pain relief, joint function and early return to daily activities

Financial and Non-Financial Relationships and Activities of Interest

None to disclose

\section{REFERENCES}

1. Victor JM, Bassens D, Bellemans J, Gürsu S, Dhollander AA, Verdonk PC. Constitutional Varus Does Not Affect Joint Line Orientation in the Coronal Plane. Clin Orthop Relat Res. 2014 Jan; 472[1]: 98-104. [DOI: 10.1007/s11999-013-2898-6].

2. Baktır A, Karaaslan F, Yurdakul E, Karaoğlu S. Mobileversus fixed-bearing total knee arthroplasty: a prospective randomized controlled trial featuring 6-10year follow-up. Acta Orthop Traumatol Turc. 2016, 50[1]: 1-9. [DOI: 10.3944/AOTT.2016.15.0120].
3. Ponzio DY, Austin MS. Metaphyseal bone loss in revision knee arthroplasty. Curr Rev Musculoskelet Med. 2015 Dec; 8[4]: 361-367. [DOI: 10.1007/s12178-015-9291-x].

4. Baldini A, Castellani L, Traverso F, Balatri A, Balato G, Franceschini V. The difficult primary total knee arthroplasty: a review. Bone Joint J. 2015 Oct;97-B[10 Suppl A]:30-9. [DOI: 10.1302/0301-620X.97B10.36920].

5. Mullaji AB, Shetty GM. Correction of Varus Deformity during TKA with Reduction Osteotomy. Clin Orthop Relat Res. 2014 Jan; 472[1]: 126-132. [DOI: 10.1007/s11999013-3077-5].

6. Hoffart HE, Dinges H, Kolbeck S, Ritschl P, Hommel H. Novel computer-assisted method for revision arthroplasty of the knee. World J Orthop. 2015 Nov 18;6 [10]: 821-8. [DOI: 10.5312/wjo.v6.i10.821].

7. Harato K, Tanikawa H, Morishige Y, Kaneda K, Niki Y. What are the important surgical factors affecting the wound healing after primary total knee arthroplasty? J Orthop Surg Res. 2016 Jan 13;11:7. [DOI: 10.1186/ s13018- 016-0340-y].

8. Bakr HMA. Total knee replacement in severe varus and flexion knee deformities using economical solutions. Egypt Ortho J. 2016; 51 (4)::329-32. [DOI: 10.4103/11101148.209001].

9. Ahn JH, Back YW. Comparative Study of Two Techniques for Ligament Balancing in Total Knee Arthroplasty for Severe Varus Knee: Medial Soft Tissue Release vs. Bony Resection of Proximal Medial Tibia. Knee Surg Relat Res. 2013 Mar; 25 (1): 13 - 8. [DOI: 10.5792/ksrr. 2013.25.1.13].

10. Abdus S, Islam A, Khan MZ, Bin Sharif S, Milon HR, Khan AS, Mahmud K. Total knee arthroplasty in patients with fixed flexion deformity. KYAMC J. 2018; 8(2): 2-7. [DOI:10.3329/kyamcj. v8i2.35695].

11. Tucker A, O'Brien S, Doran E, Gallagher N, Beverland DE. Total Knee Arthroplasty in Severe Valgus Deformity Using a Modified Technique-A 10-Year Follow-Up Study. J Arthroplasty. 2019 Jan; 34[1]:40-46. e1. [DOI:10.1016/ j.arth.2018.09.002]. 